Pacific Journal of Mathematic 


\title{
VARIETIES OF ORTHODOX BANDS OF GROUPS
}

\author{
Mario Petrich
}

The principal aim of the present work is a determination of the lattice of all varieties of semigroups in the title as a direct product of the lattice of all varieties of bands and the lattice of all varieties of groups. The paper also contains certain information concerning lattice properties of these varieties and their defining identities.

1. Introduction. A considerable amount of literature is devoted to varieties of groups, a systematic study of this subject is the book [7] by H. Neumann. Varieties of semigroups have also attracted wide attention, most of the known results are summarized in the survey article [2] by T. Evans. The lattice of all varieties of bands was determined by Birjukov [1], Gerhard [4] and Fennemore [3]; some preliminary work in this direction was first performed by Kimura [6] and the author [8].

A semigroup $S$ is completely regular if for any $a \in S$ there exists $x \in S$ such that $a=a x a, a x=x a$. It follows at once that then there exists a unique $y \in S$ such that $a=a y a, y=y a y, a y=y a$; we write $a^{-1}=y$ and observe that $S$ is a union of its (pairwise disjoint) maximal subgroups $G_{\alpha}$ and that for $a \in G_{\alpha}, a^{-1}$ is the group inverse of $a$ in $G_{\alpha}$. We consider $S$ as a universal algebra with two operations, viz., the binary operation of multiplication, and the unary operation of inversion, $a \rightarrow a^{-1}$, satisfying the identities

$$
a=a a^{-1} a, \quad a^{-1}=a^{-1} a a^{-1}, \quad a a^{-1}=a^{-1} a .
$$

The class $\mathscr{R}$ of all such universal algebras forms a variety. A semigroup $S$ in $\mathscr{R}$ is orthodox if the set $E_{s}$ of all its idempotents forms a subsemigroup. The class $\mathscr{E}$ of all orthodox semigroups in $\mathscr{R}$ is a subvariety of $\mathscr{R}$ and as such can be characterized by the identity

$$
a b=a b b^{-1} a^{-1} a b,
$$

as follows easily from ([9], IV.3.1). A semigroup $S$ in $\mathscr{R}$ in which Green's relation $\mathscr{H}$ is a congruence is a band of groups and conversely. The class $\mathscr{F}$ of all bands of groups is a subvariety of $\mathscr{R}$ and as such can be characterized by the identity 


$$
\left(a^{2} b c^{2}\right)\left(a^{2} b c^{2}\right)^{-1}=(a b c)(a b c)^{-1},
$$

as follows easily from ([9], IV.1.7).

Let $\mathscr{C}=\mathscr{E} \cap \mathscr{F}$ so that $\mathscr{C}$ is the variety of universal algebras with an associative multiplication and an inversion satisfying the identities (1), (2), (3). In fact, we can define $\mathscr{C}$, within $\mathscr{R}$, by a single identity as follows.

Proposition 1. For any $S \in \mathscr{R}$, we have that $S \in \mathscr{C}$ if and only if $S$ satisfies the identity

$$
\left(a a^{-1}\right)\left(b b^{-1}\right)=(a b)(a b)^{-1} .
$$

Proof. Necessity. Let $a, b \in S, S \in \mathscr{C}$. Then $a \mathscr{H} a a^{-1}, b \mathscr{H} b b^{-1}$ and hence $a b \mathscr{H} x a^{-1} b b^{-1}$ since Green's relation $\mathscr{H}$ is a congruence. Now $a b \mathscr{H}(a b)(a b)^{-1}$ so that $\left(a a^{-1}\right)\left(b b^{-1}\right) \mathscr{H}(a b)(a b)^{-1}$. But $E_{s}$ is a subsemigroup of $S$ and $a a^{-1}, b b^{-1},(a b)(a b)^{-1} \in E_{s}$ and thus $a a^{-1} b b^{-1}=$ $(a b)(a b)^{-1}$.

Sufficiency. Let $a, b, c \in S, S \in \mathscr{R}$ and $a \mathscr{H} b$. Then $a a^{-1}=b b^{-1}$ and hence

$$
(a c)(a c)^{-1}=\left(a a^{-1}\right)\left(c c^{-1}\right)=\left(b b^{-1}\right)\left(c c^{-1}\right)=(b c)(b c)^{-1} \text {, }
$$

i.e., $a c \mathscr{H} b c$. This shows that $\mathscr{H}$ is a right congruence. The proof that $\mathscr{H}$ is a left congruence is similar. If $e, f \in E_{S}$, then $e f=e e^{-1} f f^{-1}=$ $(e f)(e f)^{-1}$, so that $(e f)^{2}=e f$. Thus $E_{S}$ is a subsemigroup of $S$.

The class $\mathscr{B}$ of all bands is evidently a subvariety of $\mathscr{C}$ and as such can be characterized by the identity $a=a^{2}$. The class $\mathscr{G}$ of all groups is another subvariety of $\mathscr{C}$ and as such can be characterized by the identity $a a^{-1}=b b^{-1}$. If $\mathscr{V}$ is any variety of universal algebras, $\mathscr{L}(\mathscr{V})$ denotes the lattice, under inclusion, of all subvarieties of $\mathscr{V}$. One of the principal results of this paper states that

$$
\mathscr{L}(\mathscr{C}) \cong \mathscr{L}(\mathscr{B}) \times \mathscr{L}(\mathscr{G}) .
$$

We will also establish certain properties of some subvarieties of $\mathscr{C}$. In addition to the notation established above, we will use the notation, terminology and results from [9]. The meet in all our lattices will be the set theoretical intersection, the join will vary and will be denoted by $\checkmark$. For any semigroup $S$, we denote by $E_{S}$ the set of all idempotents of $S$ with the partial multiplication induced by $S$. For $e \in E_{S}, G_{e}$ denotes the maximal subgroup of $S$ having $e$ as its identity. 
2. Main result. The following lemma is crucial for a large portion of this paper.

Lemma 1. For any $\mathscr{V} \in \mathscr{L}(\mathscr{C})$, we have

$$
\mathscr{V}=(\mathscr{V} \cap \mathscr{B}) \vee(\mathscr{V} \cap \mathscr{G})
$$

Proof. Let $S \in \mathcal{V}$. According to [10], $S$ is a subdirect product of a band $B$ and a semilattice of groups $T=\cup_{\alpha \in Y} G_{\alpha}$. Since $B$ is then a homomorphic image of $S$, we have $B \in \mathscr{V} \cap \mathscr{B}$. The conjunction of ([9], IV.4.3) and ([9], III.7.2) yields that $T$ is a subdirect product of semigroups $\left\{T_{\alpha}\right\}_{\alpha \in Y}$, where either $T_{\alpha} \cong G_{\alpha}$ or $T_{\alpha} \cong G_{\alpha}^{0}$, the group $G_{\alpha}$ with a zero adjoined. Since $T$ is a homomorphic image of $S$, we have $T \in \mathscr{V}$ and thus also $G_{\alpha} \in \mathscr{V}$ for all $\alpha \in Y$.

Assume that $S$ is completely simple. Then $S \cong L \times G \times R$ where $L$ is a left zero semigroup, $G$ is a group and $R$ is a right zero semigroup, according to ([9], IV.3.3). Clearly $L \times R \in \mathscr{V} \cap \mathscr{B}$ and $G \in \mathscr{V} \cap \mathscr{G}$ and thus $S \in(\mathscr{V} \cap \mathscr{B}) \vee(\mathscr{V} \cap \mathscr{G})$.

Suppose next that $S$ is not completely simple. It is easy to see that in $S$ we can find two comparable idempotents, say $e>f$. But then $Y_{2}=\{0,1\}$, the two-element chain, must be contained in $\mathscr{V}$. Now let $G \in \mathscr{V} \cap \mathscr{G}$, and let $\rho$ be the Rees congruence on $Y_{2} \times G$ associated with the kernel $\{0\} \times G$ of $Y_{2} \times G$. It follows that

$$
G^{0} \cong\left(Y_{2} \times G\right) / \rho \in(\mathscr{V} \cap \mathscr{B}) \vee(\mathscr{V} \cap \mathscr{G})
$$

We have seen above that $T$ is a subdirect product of semigroups $T_{\alpha}$ where either $T_{\alpha} \cong G_{\alpha} \quad$ or $\quad T_{\alpha} \cong G_{\alpha}^{0}$. Consequently $T \in$ $(\mathscr{V} \cap \mathscr{B}) \vee(\mathscr{V} \cap \mathscr{G})$. Finally $S$ is a subdirect product of $B$ and $T$ and thus $S \in(\mathscr{V} \cap \mathscr{B}) \vee(\mathscr{V} \cap \mathscr{G})$.

Therefore $\mathscr{V} \subseteq(\mathscr{V} \cap \mathscr{B}) \vee(\mathscr{V} \cap \mathscr{G})$, the opposite inclusion is trivial.

LEMMA 2. Let $T$ be a completely regular semigroup which is a subdirect product of $a$ band $B$ and $a$ group $G$, and let $S$ be $a$ band and $a$ homomorphic image of $T$. Then $S$ is a homomorphic image of $B$.

Proof. Let $\varphi$ be a homomorphism of $T$ onto $S$. We may suppose that $T \subseteq B \times G$. Let $(b, g),(b, h) \in T$ and let $\bar{g}=(b, g) \varphi, \quad \bar{h}=$ $(b, h) \varphi$. Since $T$ is completely regular, we have $\left(b, h^{-1}\right) \in T$, and thus

$$
\begin{aligned}
\bar{g} & =(b, g) \varphi=\left[(b, h)\left(b, h^{-1}\right)(b, g)\right] \varphi=(b, h) \varphi\left(b, h^{-1}\right) \varphi(b, g) \varphi \\
& =(b, h) \varphi(b, g) \varphi=\bar{h} \bar{g} .
\end{aligned}
$$


A similar argument shows that $\bar{h}=\bar{h} \bar{g}$ and thus $\bar{g}=\bar{h}$. It follows that the mapping $\psi$ defined by

$$
\psi: b \rightarrow(b, g) \varphi \quad \text { if } \quad(b, g) \in T \quad(b \in B)
$$

is single-valued, and thus evidently a homomorphism of $B$ onto $S$.

LEMMA 3. Let T be a semigroup which is a subdirect product of a band $B$ and a semigroup $C$, and let $S$ be a left cancellative semigroup and a homomorphic image of $T$. Then $S$ is a homomorphic image of $C$.

Proof. Let $\varphi$ be a homomorphism of $T$ onto $S$, and suppose that $T \subseteq B \times C$. Let $(a, c),(b, c) \in T$. Then

$$
\left(a, c^{2}\right)(b, c)=\left(a b, c^{3}\right)=\left((a b) b, c^{3}\right)=\left(a b, c^{2}\right)(b, c)
$$

where $\left(a, c^{2}\right),\left(a b, c^{2}\right) \in T$ and thus

$$
[(a, c) \varphi]^{2}=\left(a, c^{2}\right) \varphi=\left(a b, c^{2}\right) \varphi=(a, c) \varphi(b, c) \varphi .
$$

Left cancellation in $S$ now implies that $(a, c) \varphi=(b, c) \varphi$. It follows that the mapping $\psi$ defined by

$$
\psi: c \rightarrow(a, c) \quad \text { if } \quad(a, c) \in T \quad(c \in C)
$$

is single-valued, and thus evidently a homomorphism of $C$ onto $S$.

THEOREM. The mapping $\chi$ defined by

$$
\chi: \mathscr{V} \rightarrow(\mathscr{V} \cap \mathscr{B}, \mathscr{V} \cap \mathscr{G}) \quad(\mathscr{V} \in \mathscr{C})
$$

is an isomorphism of $\mathscr{L}(\mathscr{C})$ onto $\mathscr{L}(\mathscr{B}) \times \mathscr{L}(\mathscr{G})$.

Proof. It is obvious that $\chi$ is inclusion preserving. Let $\mathscr{V}^{\prime} \in$ $\mathscr{L}(\mathscr{B})$ and $\mathscr{V}^{\prime \prime} \in \mathscr{L}(\mathscr{G})$, and let $\mathcal{V}=\mathscr{V}^{\prime} \vee \mathscr{V}^{\prime \prime}$. Then

$$
\mathscr{V} \cap \mathscr{B}=\left(\mathscr{V}^{\prime} \vee \mathscr{V}^{\prime \prime}\right) \cap \mathscr{B} \supseteq \mathscr{V}^{\prime} \cap \mathscr{V}=\mathscr{V}^{\prime \prime}
$$

In order to establish the opposite inclusion, we let $S \in \mathscr{V} \cap \mathscr{B}$. In view of ([5], §23, Theorem 3), there exist $B \in \mathscr{V}^{\prime}, G \in \mathscr{V}^{\prime \prime}$, a completely regular semigroup $T$ which is a subdirect product of $B$ and $G$, and a homomorphism $\varphi$ of $T$ onto $S$. Hence by Lemma $2, S$ is a homomorphic image of $B$ and thus $S \in \mathscr{V}^{\prime}$. Consequently $\mathscr{V} \cap \mathscr{B}=\mathscr{V}^{\prime} . \quad$ A 
similar argument, using Lemma 3 , shows that $\mathscr{V} \cap \mathscr{G}=\mathscr{V}^{\prime \prime}$. It follows that $\mathscr{V} \in \mathscr{C}$ and that $\mathscr{V} \chi=\left(\mathscr{V}^{\prime}, \mathscr{V}^{\prime \prime}\right)$, proving that $\chi$ maps $\mathscr{L}(\mathscr{C})$ onto $\mathscr{L}(\mathscr{B}) \times \mathscr{L}(\mathscr{G})$. Now Lemma 1 easily implies that $\chi$ is one-to-one and that $\chi^{-1}$ is inclusion preserving. Therefore $\chi$ is a lattice isomorphism.

Corollary. For any $S \in \mathscr{C}, \mathscr{V}^{\prime} \in \mathscr{L}(\mathscr{B}), \mathscr{V}^{\prime \prime} \in \mathscr{L}(\mathscr{G})$, we have $S \in \mathscr{V}^{\prime} \vee \mathscr{V}^{\prime \prime}$ if and only if $E_{s} \in \mathscr{V}^{\prime}$ and $G_{e} \in \mathscr{V}^{\prime \prime}$ for all $e \in E_{s}$.

Proof. This follows without difficulty from the proof of the theorem and the proof of Lemma 1.

3. Further results. We consider first the following problem: if $\mathscr{V}^{\prime} \in \mathscr{L}(\mathscr{B})$ and $\mathscr{V}^{\prime \prime} \in \mathscr{L}(\mathscr{G})$ are given by their defining identities, can we set up a system of defining identities for $\mathscr{V}^{\prime} \vee \mathscr{V}^{\prime \prime}$ ? We now proceed to describe such a system.

Let $u=v$ be an identity on $\mathscr{B}$. Substitute every variable $x$ that occurs in $u=v$ by $x x^{-1}$. We then obtain an identity on $\mathscr{C}$, to be denoted by $\bar{u}=\bar{v}$.

Let $w=z$ be an identity on $\mathscr{G}$. We may suppose that both $w$ and $z$ contain the same set $\left\{x_{1}, x_{2}, \cdots, x_{n}\right\}$ of variables. Consider $w=z$ as an identity on $\mathscr{C}$, and let $e=\left(x_{1}, x_{2} \cdots x_{n}\right)\left(x_{1} x_{2} \cdots x_{n}\right)^{-1}$. Substitute each occurrence of $x_{i}$ in $w=z$ by $e x_{i} e$. We then obtain an identity on $\mathscr{C}$, to be denoted by $\hat{w}=\hat{z}$. Note that $e$ depends on the choice of writing the variables, but any single choice will do.

PROPOSITION 2. Let $\mathscr{V}^{\prime}$ (resp. $\mathscr{V}^{\prime \prime}$ ) be the variety of bands (resp. groups) defined by a system of identities $\left\{u_{\alpha}=v_{\alpha}\right\}$ (resp. $\left\{w_{\beta}=z_{\beta}\right\}$ ). Then $\mathscr{V}^{\prime} \vee \mathscr{V}^{\prime \prime}$ can be defined by the system $\left\{\bar{u}_{\alpha}=\bar{v}_{\alpha}, \hat{w}_{\beta}=\right.$ $\left.\hat{z}_{\beta}\right\}$.

Proof. By the above corollary, $\mathscr{V}=\mathscr{V}^{\prime} \vee \mathscr{V}^{\prime \prime}$ consists of all $S \in \mathscr{C}$ for which $E_{S} \in \mathscr{V}^{\prime}$ and $G_{e} \in \mathscr{V}^{\prime \prime}$ for all $e \in E_{S}$. Let $S \in \mathscr{V}$. Then $E_{S}$ satisfies $u_{\alpha}=v_{\alpha}$ and hence $S$ satisfies $\bar{u}_{\alpha}=\bar{v}_{\alpha}$. Next consider $w_{\beta}=$ $z_{\beta}$. Let $\left\{x_{1}, x_{2}, \cdots, x_{n}\right\}$ be the set of variables occurring in $w_{\beta}=z_{\beta}$. For any $a \in S$, we denote by $N_{a}$ the class of the least semilattice congruence on $S$ containing $a$. Let $a_{1}, a_{2}, \cdots, a_{n} \in S$ and $e=$ $\left(a_{1} a_{2} \cdots a_{n}\right)\left(a_{1} a_{2} \cdots a_{n}\right)^{-1}$. Then for any $1 \leqq i \leqq n$, $e a_{i} e \in G_{e}$ since

$$
e a_{i} e \in N_{e a_{i} e}=N_{e} N_{a_{i}} N_{e}=N_{e}
$$

and $N_{e}$ is completely simple. Observing that each $G_{e}$ satisfies the identity $w_{\beta}=z_{\beta}$, we deduce that $S$ satisfies the identity $\hat{w}_{\beta}=$ $\hat{z}_{\beta}$. Consequently each $S \in \mathscr{V}$ satisfies all the identities $\bar{u}_{\alpha}=\bar{v}_{\alpha}, \hat{w}_{\beta}=$ $\hat{z}_{\beta}$. 
Conversely, let $S \in \mathscr{C}$ satisfy all the identities $\bar{u}_{\alpha}=\bar{v}_{\alpha}, \hat{w}_{\beta}=$ $\hat{z}_{\beta}$. Then $E_{S}$ satisfies each $u_{\alpha}=v_{\alpha}$ so that $E_{S} \in \mathscr{V}^{\prime}$. Further, for every $e \in E_{S}, G_{e}$ satisfies each $\hat{w}_{\beta}=\hat{z}_{\beta}$, and hence also $w_{\beta}=z_{\beta}$ since $G_{e}$ has only one idempotent. Thus $G_{e} \in \mathscr{V}^{\prime \prime}$. By the above corollary we conclude that $S \in \mathscr{V}^{\prime} \vee \mathscr{V}^{\prime \prime}=\mathscr{V}$.

For example, if $\mathscr{V}^{\prime}$ is the variety of all rectangular bands and $\mathscr{V}^{\prime \prime}$ the variety of all groups, then $\mathscr{V}=\mathscr{V}^{\prime} \vee \mathscr{V}^{\prime \prime}$ can be defined by the identity $x x^{-1}=x x^{-1} y y^{-1} x x^{-1}$, which is evidently equivalent to $x^{2}=x y y^{-1} x$. This identity defines the subvariety of rectangular groups.

As another example, we may take $\mathcal{V}^{\prime}$ to be the variety of all bands and $\mathscr{V}^{\prime \prime}$ the variety of all abelian groups. Then $\mathscr{V}=\mathscr{V}^{\prime} \vee \mathscr{V}^{\prime \prime}$ can be defined by the identity $x x^{-1} x y x x^{-1}=x x^{-1} y x x x^{-1}$, which is evidently equivalent to $x^{2} y x=x y x^{2}$. This identity defines the subvariety of orthodox bands of abelian groups.

We consider next the following question: which subvarieties of $\mathscr{C}$ are simultaneously subvarieties of the variety $\mathscr{S}$ of all semigroups? For an identity $u=v$ on $\mathscr{S}$, we denote by $[u=v]$ the variety of semigroups defined by $u=v$. If $x$ is an element of a semigroup $S,\langle x\rangle$ denotes the cyclic subsemigroup of $S$ generated by $x$.

Proposition 3. The following conditions on a subvariety $\mathscr{V}$ of $\mathscr{C}$ are equivalent.

(i) $\quad \mathscr{V} \in \mathscr{L}(\mathscr{S})$.

(ii) $\mathscr{V} \subseteq\left[x=x^{n}\right]$ for some integer $n>1$.

(iii) $\mathscr{V} \cap \mathscr{G} \subseteq\left[x=x^{n}\right]$ for some integer $n>1$.

(iv) $\mathscr{V} \cap \mathscr{G} \in \mathscr{L}(\mathscr{P})$.

Proof. (i) implies (ii). Let $x \in S$ and $S \in \mathscr{V}$. Then $\langle x\rangle \in \mathscr{V}$ since $\mathscr{V} \in \mathscr{L}(\mathscr{S}$. But then $\mathscr{V} \in \mathscr{L}(\mathscr{C})$ implies that $\langle x\rangle \in \mathscr{C}$ which is possible only if $\langle x\rangle$ is a finite group. Hence $x=x^{n}$ for some $n>$ 1. Assume that the set

$$
\left\{n(x) \mid x^{n(x)}=x, \quad x \in S, \quad S \in \mathscr{V}\right\}
$$

is unbounded. Hence there exists an infinite sequence $\left\langle x_{1}\right\rangle,\left\langle x_{2}\right\rangle, \cdots$ of cyclic semigroups such that $n\left(x_{1}\right)<n\left(x_{2}\right)<\cdots$. The element $\left(x_{i}\right)$ of the direct product $S=\prod_{i=1}^{\infty}\left\langle x_{i}\right\rangle$ is clearly of infinite order. Since $S \in \mathscr{V}$, this contradicts to. what we have proved above. Thus there exists $n>1$ such that $\mathscr{V} \subseteq\left[x=x^{n}\right]$.

Items (ii) and (iii) are obviously equivalent. Item (ii) implies item (i) since with $x=x^{n}$ in any semigroup $S, x^{n-1}$ is the identity of the cyclic 
group generated by $x$. The equivalence of (iii) and (iv) follows similarly as the equivalence of (i) and (ii).

We now elucidate another relationship between $\mathscr{L}(\mathscr{C})$ and $\mathscr{L}(\mathscr{Y})$.

Proposition 4. Let $\mathscr{V} \in \mathscr{L}(\mathscr{C})$ and $\mathcal{U} \in \mathscr{L}\left(\mathscr{S}_{1}\right.$. Then $\mathcal{U} \cap \mathscr{C}=\mathscr{V}$ if and only if $\mathcal{U} \cap \mathscr{G}=\mathcal{V} \cap \mathscr{G}$ and $\mathcal{U} \cap \mathscr{B}=\mathscr{V} \cap \mathscr{B}$.

Proof. If $\mathcal{U} \cap \mathscr{C}=\mathscr{V}$, then

$$
\mathcal{U} \cap \mathscr{G}=\mathscr{U} \cap(\mathscr{G} \cap \mathscr{C})=(\mathscr{U} \cap \mathscr{C}) \cap \mathscr{G}=\mathscr{V} \cap \mathscr{G}
$$

and analogously $\mathcal{U} \cap \mathscr{B}=\mathscr{V} \cap \mathscr{B}$.

Conversely, suppose that $\mathscr{u} \cap \mathscr{G}=\mathscr{V} \cap \mathscr{G}$ and $\mathscr{U} \cap \mathscr{B}=$ $\mathscr{V} \cap \mathscr{B}$. The join in $\mathscr{C}$ will be now denoted by $\stackrel{c}{\vee}$ and the join in $\mathscr{S}$ by v. Using Lemma 1, we obtain

$$
(\mathscr{V} \cap \mathscr{B}) \stackrel{c}{\vee}(\mathcal{U} \cap \mathscr{G}=(\mathscr{V} \cap \mathscr{B}) \stackrel{c}{\vee}(\mathscr{V} \cap \mathscr{G})=\mathscr{V}
$$

so that

$$
\mathscr{V} \subseteq((\mathscr{V} \cap \mathscr{B}) \stackrel{s}{\vee} \mathcal{U}) \cap \mathscr{C}
$$

In order to establish the opposite inclusion, we first let $G \in$ $\left((\mathscr{V} \cap \mathscr{B}) \vee^{s} \mathcal{U}\right) \cap \mathscr{G}$. In view of ([5], §23, Theorem 3$)$, there exist $B \in \mathscr{V} \cap \mathscr{B}, C \in \mathcal{U}$, a subdirect product $T$ of $B$ and $C$ and a homomorphism of $T$ onto $G$. By Lemma $3, G$ is a homomorphic image of $C$ and thus $G \in \mathcal{U}$. Consequently $G \in \mathcal{U} \cap \mathscr{G}=\mathcal{V} \cap \mathscr{G}$. Next let $B \in((\mathscr{V} \cap \mathscr{B}) \stackrel{s}{\vee} \mathcal{U}) \cap \mathscr{B}$. Then

$$
B \in((\mathcal{U} \cap \mathscr{B}) \stackrel{s}{\vee} \mathcal{U}) \cap \mathscr{B}=\mathcal{U} \cap \mathscr{B}=\mathscr{V} \cap \mathscr{B} .
$$

It follows that

$$
[((\mathscr{V} \cap \mathscr{B}) \stackrel{s}{\vee} \mathcal{U}) \cap \mathscr{B}] \stackrel{c}{\vee}[((\mathscr{V} \cap \mathscr{B}) \stackrel{s}{\vee} \mathcal{U}) \cap \mathscr{G}] \subseteq \mathscr{V}
$$


and thus by Lemma 1, we have

$$
((\mathscr{V} \cap \mathscr{B}) \stackrel{s}{\vee} \mathcal{U}) \cap \mathscr{C} \subseteq \mathscr{V} .
$$

The conjunction of (4) and (5) yields

$$
((\mathscr{V} \cap \mathscr{B}) \stackrel{s}{\vee}) \cap \mathscr{C}=\mathscr{V},
$$

where $(\mathscr{V} \cap \mathscr{B}) \stackrel{s}{\vee} \mathcal{U} \in \mathscr{L}(\mathscr{S}$. But $\mathscr{V} \cap \mathscr{B}=\mathscr{U} \cap \mathscr{B}$ implies that $(\mathscr{V} \cap \mathscr{B}) \dot{v} \mathcal{U}=\mathscr{U}$ which by (6) gives $\mathcal{U} \cap \mathscr{C}=\mathscr{V}$, as required.

Note that Proposition 4 implies the following statement: if $\mathscr{V} \in$ $\mathscr{L}(\mathscr{C})$ and $\mathscr{V}=\mathcal{U} \cap \mathscr{C}$ for some $\mathcal{U} \in \mathscr{L}\left(\mathscr{S}_{1}\right.$, then $\mathscr{V} \cap \mathscr{G}=\mathcal{U} \cap \mathscr{G}$. A converse of this statement can be phrased thus: If $\mathscr{V}^{\prime} \in \mathscr{L}(\mathscr{B})$ and $u \in \mathscr{L}\left(\mathscr{S}_{1}\right.$, does there exist $\mathcal{V} \in \mathscr{L}\left(\mathscr{S}_{1}\right.$ ) such that

$$
\mathscr{V}^{\prime} \stackrel{c}{\vee}(\mathcal{U} \cap \mathscr{G}=\mathscr{V} \cap \mathscr{C} ?
$$

An answer to this question is open. However, we have the following simple result. For any class $\mathscr{D}$ of semigroups, let $\mathscr{D}_{\mathscr{S}}$ denote the variety of semigroups generated by $\mathscr{D}$.

Proposition 5. Let $\mathscr{V} \in \mathscr{L}(\mathscr{C})$. Then there exists $\mathscr{U} \in \mathscr{L}(\mathscr{S}$, such that $\mathscr{V}=\mathcal{U} \cap \mathscr{C}$ if and only if $\mathscr{V}_{\mathscr{\varphi}} \cap \mathscr{C}=\mathscr{V}$.

Proof. Necessity. Let $S \in \mathscr{V}_{\mathscr{S}} \cap \mathscr{C}$. According to ([5], §23, Theorem 3) $S$ is a homomorphic image of a subsemigroup $T$ of some semigroup $H$ in $\mathscr{V}$. It follows that $H \in \mathcal{U} \cap \mathscr{C}$ and thus $T \in \mathcal{U}$ and hence also $S \in \mathcal{U}$. Consequently $S \in \mathcal{U} \cap \mathscr{C}=\mathscr{V}$. This proves that $\mathscr{V}_{\mathscr{S}} \cap \mathscr{C} \subseteq \mathscr{V}$, the opposite inclusion is trivial.

Sufficiency. Take $U=\mathscr{V}_{\varphi}$.

For example, for $\mathscr{V}=\mathscr{G}$ or the varieties of all left, right or rectangular groups, we have the inequality $\mathscr{V}_{\mathscr{S}} \cap \mathscr{C} \neq \mathscr{V}$. This shows, in particular, that these subvarieties of $\mathscr{C}$ cannot be defined, within $\mathscr{C}$, by semigroup identities alone. To see this, let $G$ be the additive group of all integers, $T$ the subsemigroup of $G$ consisting of all nonnegative integers, $S$ the multiplicative semigroup $\{0,1\}$, and $\varphi$ be the mapping defined by: $0 \varphi=1, n \varphi=0$ for all $n \in T, n \neq 0$. Then $S \notin \mathscr{V}$ and $S \in \mathscr{V}_{\mathscr{S}} \cap \mathscr{C}$. 


\section{REFERENCES}

1. A. P. Birjukov, Varieties of idempotent semigroups, Algebra i Logika, 9 (1970), 255-273 (in Russian); Transl. Algebra and Logic, Consult. Bureau, 9 (1970), 153-164.

2. T. Evans, The lattice of semigroup varieties, Semigroup Forum, 2 (1971), 1-43.

3. C. F. Fennemore, All varieties of bands, Math. Nachr., 48 (1971); I:237-252, II:253-262.

4. J. A. Gerhard, The lattice of equational classes of idempotent semigroups, J. Algebra, 15 (1970), 195-224.

5. G. Grätzer, Universal Algebra, Van Nostrand, Princeton, 1968.

6. N. Kimura, Note on idempotent semigroups. IV. Identities of three variables, Proc. Japan Acad., 24 (1958), 121-123.

7. H. Neumann, Varieties of groups, Erg. Math. u.i. Grenzg., Vol. 37, Springer, Berlin, 1967.

8. M. Petrich, A construction and a classification of bands, Math. Nachr., 48 (1971), 263-274.

9. Introduction to Semigroups, Merill, Columbus, 1973.

10. B. M. Schein, A note on radicals in regular semigroups, Semigroup Forum, 3 (1971), 84-85.

Received March 27, 1973.

The Pennsylvania State University 



\section{CONTENTS}

Zvi Artstein and John A. Burns, Integration of compact set-valued functions

J. A. Beachy and W. D. Blair, Rings whose faithful left ideals are cofaithful

Mark Benard, Characters and Schur indices of the unitary reflection group $[321]^{3}$

H. L. Bentley and B. J. Taylor, Wallman rings ............................. 15

E. Berman, Matrix rings over polynomial identity rings II ............... 37

Simeon M. Berman, A new characterization of characteristic functions of absolutely continuous distributions ........................ 323

Monte B. Boisen, Jr. and Philip B. Sheldon, Pre-Prüfer rings ............ 331

A. K. Boyle and K. R. Goodearl, Rings over which certain modules are injective

J. L. Brenner, R. M. Crabwell and J. Riddell, Covering theorems for finite nonabelian simple groups. $V$

H. H. Brungs, Three questions on duo rings .................................... 345

Iracema M. Bund, Birnbaum-Orlicz spaces of functions on groups ....351

John D. Elwin and Donald R. Short, Branched immersions between 2-manifolds of higher topological type

J. K. Finch, The single valued extension property on a Banach space ................................................................................. 61

J. R. Fisher, A Goldie theorem for differentiably prime rings ............ 71

Eric M. Friedlander, Extension functions for rank 2, torsion free abelian groups ...................................................................... 371

J. Froemke and R. Quackenbusch, The spectrum of an equational class of groupoids

B. J. Gardner, Radicals of supplementary semilattice sums of

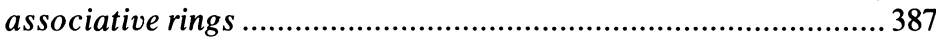

Shmuel Glasner, Relatively invariant measures ...............................393

G. R. Gordh, Jr. and Sibe Mardešić, Characterizing local connectedness in inverse limits...

S. Graf, On the existence of strong liftings in second countable topological spaces

S. Gudder and D. Strawther, Orthogonally additive and orthogonally increasing functions on vector spaces ........................................427

F. Hansen, On one-sided prime ideals .......................................... 79

D. J. Hartfiel and C. J. Maxson, A characterization of the maximal monoids and maximal groups in $\beta x$.

Robert E. Hartwig and S. Brent Morris, The universal flip matrix and the generalized faro-shuffle 


\section{Pacific Journal of Mathematics}

Vol. 58, No. 1

March, 1975

John Allen Beachy and William David Blair, Rings whose faithful left ideals are cofaithful .................................... 1

Herschel Lamar Bentley and Barbara June Taylor, Wallman rings ........ 15

Elizabeth Berman, Matrix rings over polynomial identity rings. II ...... 37

Ann K. Boyle and Kenneth R. Goodearl, Rings over which certain modules are injective ................................. 43

J. L. Brenner, Robert Myrl Cranwell and James Riddell, Covering theorems for finite nonabelian simple groups. $V \ldots \ldots \ldots \ldots \ldots \ldots \ldots \ldots \ldots$

James Kenneth Finch, The single valued extension property on a Banach

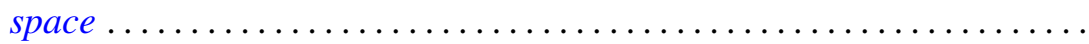

John Robert Fisher, A Goldie theorem for differentiably prime rings........ Friedhelm Hansen, On one-sided prime ideals .................... Jon Craig Helton, Product integrals and the solution of integral equations..........................................

Barry E. Johnson and James Patrick Williams, The range of a normal

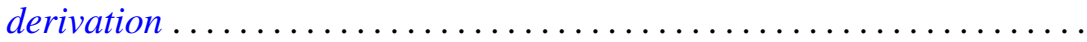

Kurt Kreith, A dynamical criterion for conjugate points ............ 123

Robert Allen McCoy, Baire spaces and hyperspaces .............. 133

John McDonald, Isometries of the disk algebra ................ 143

H. Minc, Doubly stochastic matrices with minimal permanents ......... 155

Shahbaz Noorvash, Covering the vertices of a graph by vertex-disjoint paths. ...

Theodore Windle Palmer, Jordan *-homomorphisms between reduced Banach*-algebras

Donald Steven Passman, On the semisimplicity of group rings of some

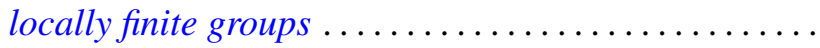

Mario Petrich, Varieties of orthodox bands of groups .

Robert Horace Redfield, The generalized interval topology on distributive

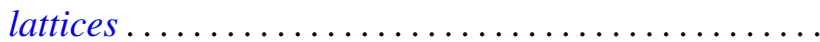

James Wilson Stepp, Algebraic maximal semilattices .... . .

Patrick Noble Stewart, A sheaf theoretic representation of rings with Boolean orthogonalities ........................

Ting-On To and Kai Wing Yip, A generalized Jensen's inequality......... 255

Arnold Lewis Villone, Second order differential operators with self-adjoint

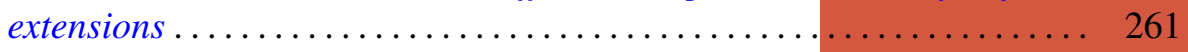

Martin E. Walter, On the structure of the Fourier-Stieltjes algebra ....... 267

John Wermer, Subharmonicity and hulls .................... 283

Edythe Parker Woodruff, A map of $E^{3}$ onto $E^{3}$ taking no disk onto a

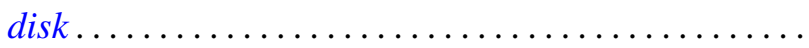

